\title{
Cinématique et vitesse d'évolution des nappes superficielles : une simulation numérique
}

\author{
Kinematic and velocity of thrust sheets emplacement : \\ a numerical modeling
}

\author{
J.-L. MUGNIER \\ Institut Dolomieu et URA CNRS $n^{\circ} 69^{\circ}$ \\ L. ENDIGNOUX \\ Institut Français du Pétrole**
}

Rev. Franç. Géotech. n 56, pp. 23-32 (juillet 1991)

\section{Résumé}

Le déplacement et la déformation des nappes peuvent être décrits, en première approximation, par des algorithmes cinématiques simples, basés sur l'hypothèse suivante: le corps de la nappe se déforme par plissement lors du déplacement de la nappe, et la localisation des plis est liée aux irrégularités de la surface basale. Un programme informatique a été écrit, permettant de simuler, grâce à ces algorithmes, le déplacement des nappes. Son application à l'étude de la cinématique et de la vitesse de formation des Apennins est ici présentée. Les couplages possibles entre modèles cinématiques, modèles de flexion lithosphérique et modèles thermiques sont illustrés.

\section{Abstract}

Kinematical algorithms are used to describe the displacement and the deformation of a nappe. They assume that the thrust sheet is deformed by folding during the displacement of the nappe, and that the location of the folds is linked to the variations in the dip of the sole fault. A computer code has been written and has been used to study the Apennin thrust belt. The relations between the kinetic of thrust motion, the flexured isostatic equilibrium and the thermal state are considered. 
Dès la fin du $19^{e}$ siècle, les nappes ont été définies en tant que structures géologiques. En effet, il était nécessaire de trouver une explication cinématique à la superposition anormale, sur une même verticale, de terrains anciens reposant sur des terrains plus jeunes. Le concept de nappe a donc été proposé pour expliquer des déplacements horizontaux pluri-kilométriques de volumes rocheux très importants (plusieurs kilomètres cubes).

Les méthodes ici présentées (voir SUPPE, 1984 , pour plus de détails) s'appliquent à des structures situées dans la partie superficielle (dans les 5 à 10 premiers kilomètres) de la croûte terrestre. Dans ce domaine une discontinuité cinématique - la surface de chevauchement - sépare lallochtone (les roches déplacées) de l'autochtone (les roches en place). L'estimation de l'ampleur des déplacements sur cette discontinuité repose sur l'analyse des bilans de grandeurs géométriques (volume, longueur, épaisseur de couches...) (fig. 1).

On peut alors réaliser des coupes géologiques pour lesquelles ce bilan apparaît comme équilibré (MUGNIER, 1984). Dans cet article, est présentée une simulation numérique du déplacement des nappes permettant d'informatiser la construction de coupes équilibrées. Outre la description de la simulation et de son application à des structures réelles, sa signification physique et son utilisation sont discutées.
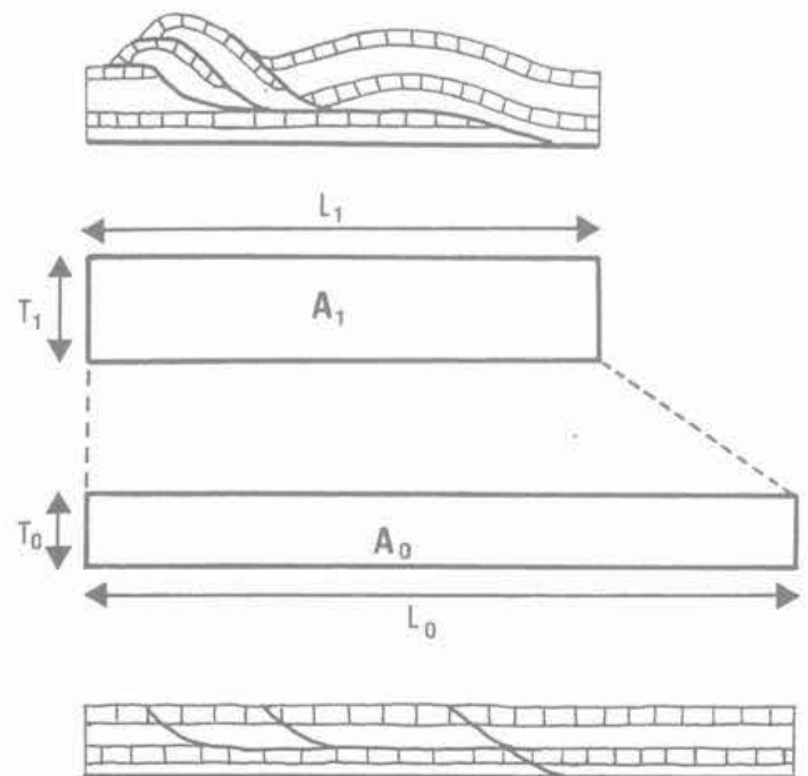

Fig. 1. - Bilan des surfaces et des longueurs. La déformation est isovolumique, et en coupe $A_{0}=A_{1}$. ( $A_{0}$ : aire initiale; $A_{1}$ : aire finale). La longueur des horizons compétents est conservée : $L_{0}-L_{1}\left(L_{0}\right.$ : longueur initiale, $L_{1}$ : longueur finale) exprime les flèches de chevauchements, et les déplacements relatifs entre les différentes écailles.

Fig. 1. - Line length and area balancing. The bulk deformation preserves the volume, and in a section $A_{0}=A_{1}$. The length of competent strata is preserved: $L_{0}-L_{1}$ is the displacement at the trailing edge of the thrust sheets.

\section{LA MÉTHODE DE SIMULATION}

\subsection{Trajectoire de la surface de chevauchement}

Dans le domaine externe d'une chaîne de montagnes, la tectonique affecte un empilement de quelques kilomètres d'épaisseur de sédiments hétérogènes, dont le litage induit une anisotropie des propriétés mécaniques. La déformation d'ensemble des couches sédimentaires lors de la formation des nappes s'effectue par l'apparition de discontinuités cinématiques (failles) dont la trajectoire est guidée par l'hétérogénéité de la pile : failles parallèles aux couches $*$ faibles $»$ (zones plates) et obliques, souvent avec un pendage de $30^{\circ}$ par rapport aux couches plus compétentes (BOYER et ELLIOTT, 1982). Du point de vue mécanique, on peut considérer que les couches * faibles * fluent alors que les couches compétentes ont un comportement fragile dont le seuil de rupture est régi par un critère de Mohr-Coulomb.

Pour avoir un état de contrainte cohérent à la fois avec un fluage des couches «faibles » et un glissement le long de fractures orientées à environ $30^{\circ}$ par rapport au plan de stratification, il faut envisager une direction principale de compression voisine de l'horizontale et un seuil de fluage très bas par rapport au seuil de fracturation (fig. 2).

\subsection{Déformation de l'allochtone}

L'allochtone se déplace le long de la surface de chevauchement en se déformant pour s'adapter aux irrégularités de la surface de base. Certains glaciers fournissent une bonne analogie pour comprendre ce pro-

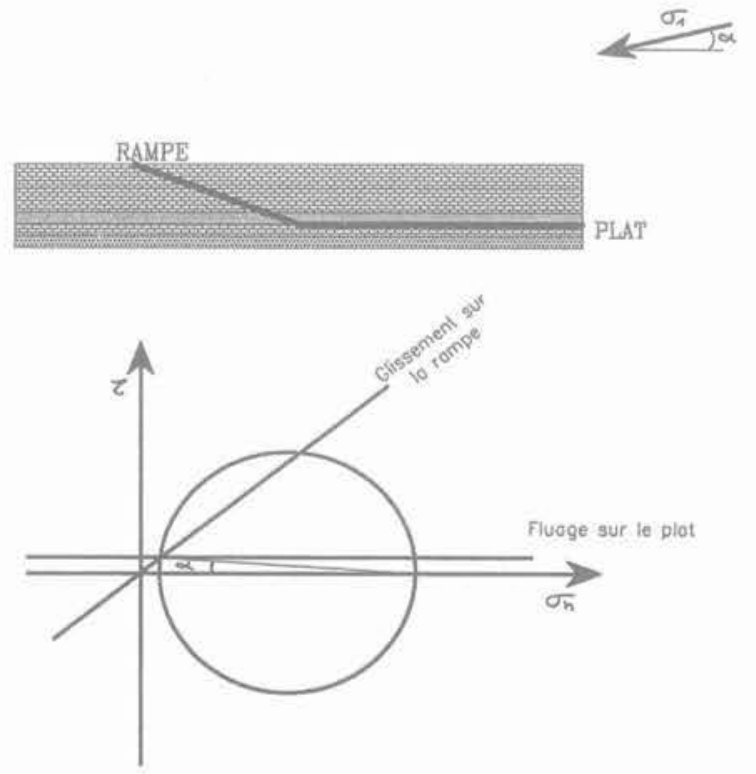

Fig. 2. - Trajectoire en plat et rampe d'une surface de chevauchement dans une pile sédimentaire et état de contrainte permettant le déplacement le long de la surface de chevauchement.

Fig. 2. - Ramp-flat trajectory of a thrust and stress field during the displacement of the thrust sheet 
cessus. Au niveau d'une rupture de pente, le glacier moule cette dernière lors de son déplacement.

La déformation du glacier a à la fois un caractère fragile - apparition de crevasses - et également ductile - fluage et plissement d'ensemble.

Pour simuler ce phénomène d'une façon simple on utilise différents modèles de plissement (MORETTI et al., 1989). Deux d'entre eux sont plus particulièrement utilisés :

- le modèle de plis en kink (SUPPE, 1983) dans lesquels les strates sont des lignes de glissement, et dans lesquels la perturbation liée aux irrégularités de la surface de base se localise dans une surface axiale bissectrice des flancs du pli (fig. 3A); dans ce cas P'épaisseur est conservée perpendiculairement à la surface de base et l'épaisseur verticale est donc augmentée ;

- les plis semblables dans lesquels les épaisseurs verticales sont conservées (JONES et LINSSER, 1986) (fig. 3B)

La géométrie des plis dans la nature est bien entendu plus complexe que ces modèles simplistes; et a été analysée en détail par de nombreux auteurs. Il apparaît cependant que pour des formations calcaires bien litées, le modèle des plis en kink est une bonne approximation, et c'est celle que nous utiliserons principalement.

Dans ces modèles de plissement, on est conduit à définir deux types de plans axiaux, ceux qui se déplacent avec la nappe, et donc fixes par rapport à celleci, et ceux fixes par rapport à l'autochtone qui voient passer l'allochtone (CRANE, 1987) (fig. 4).
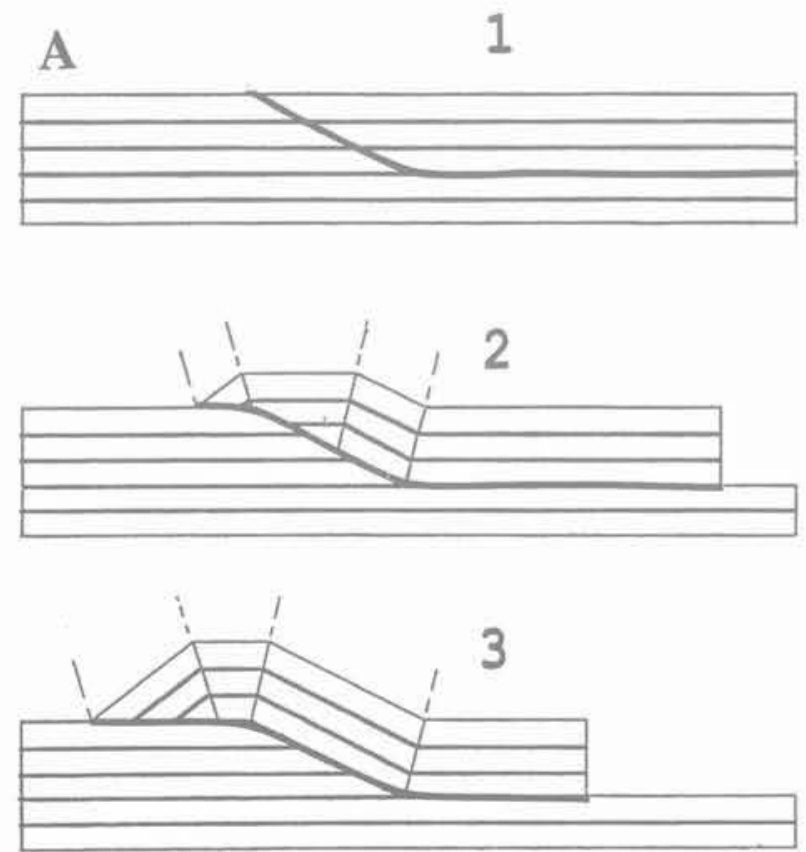

Une des implications de ces modèles est qu'ils prédisent d'éventuelles accentuations ou atténuations de la forme des plis dans la nappe. Ceci a un intérêt pétrolier immédiat : un anticlinal est un piège potentiel pour le pétrole avec une fermeture basse définie par les limites latérales de l'anticlinal. Alors que dans un modèle de plissement par flambage la fermeture est définie dès les premiers stades de la compression, dans les modèles de plissement à l'aplomb des rampes la fermeture peut être réalisée très tardivement (fig. 5).

\subsection{Application informatique}

Des algorithmes simples ont été écrits pour simuler le déplacement des nappes dans un plan de coupe parallèle à leur direction de mouvement. Il s'agit de modèles de déformation dit " direct " dans la mesure où l'on simule les déformations dans l'ordre où elles se produisent. Les paramètres introduits dans le programme "CICERON * (Cinématique des Chevauchements Equilibrés en Coupes Reconstruites dans l'Ordre et Numériquement) ici utilisé, sont :

- le nombre de failles ;

- la trajectoire de chacune des failles dans la pile sédimentaire avant déformation. Cette trajectoire est considérée comme constituée par une succession de segments de droite ;

- le déplacement le long de chaque faille (sa valeur étant définie à l'arrière du système déplacé) ;

- la séquence de fonctionnement des différentes failles.

\section{B}
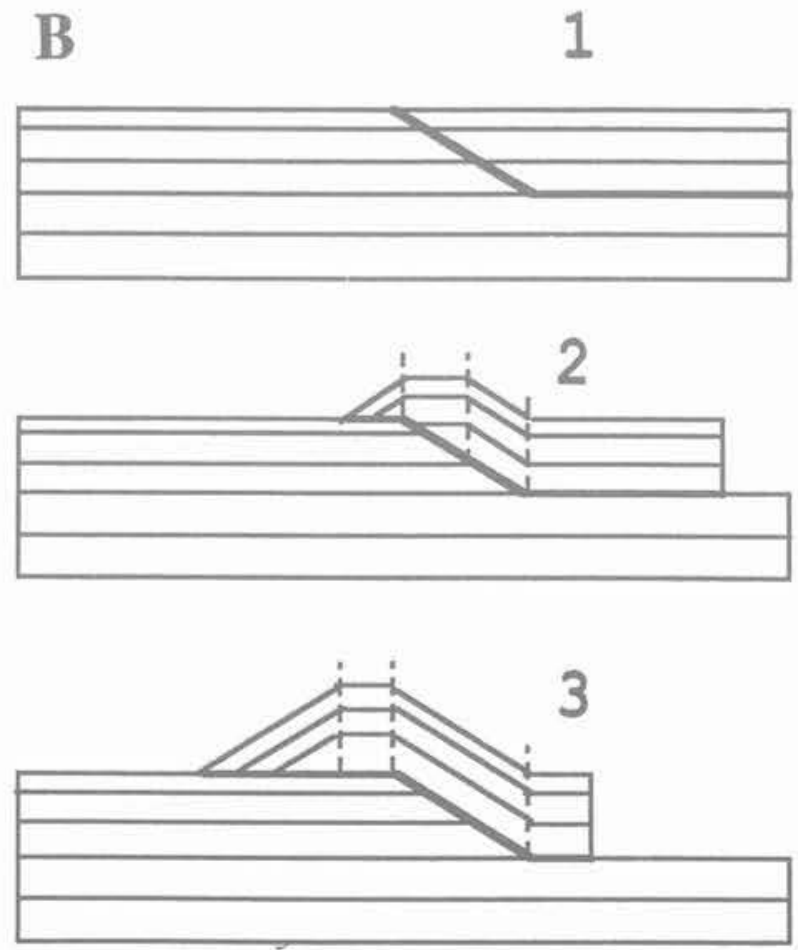

Fig. 3. - Développement de plis A; en kink, et B: de plis semblables (à droite) à l'aplomb d'une rampe. Fig. 3. - A: Kink fold and B: vertical simple shear fold at the hanging-wall of a ramp. 

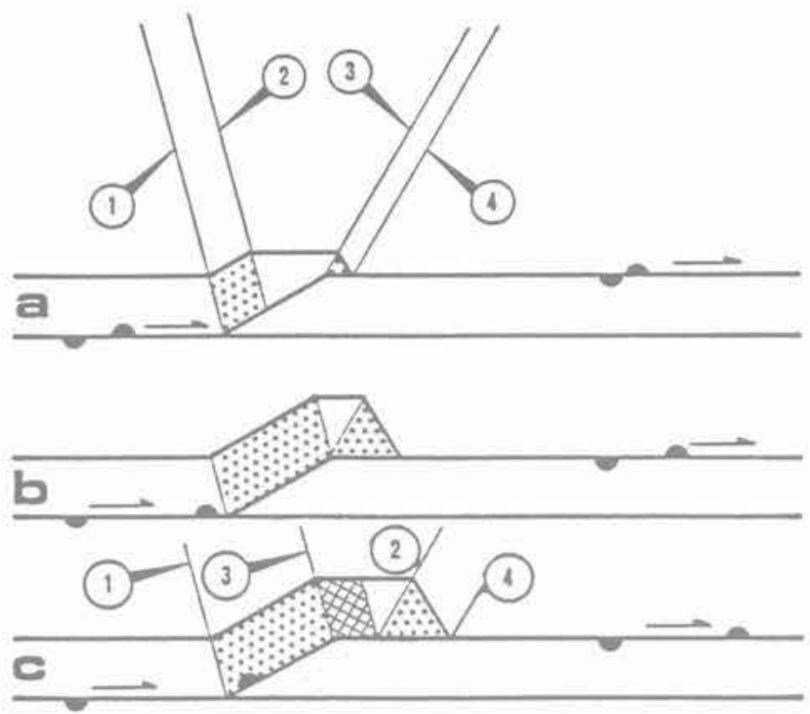

Fig. 4. - Les plans axiaux mobiles $(2,4)$ et fixes $(1,3)$ par rapport à l'autochtone lors de la formation d'un pli de rampe.

Fig. 4. - Migrating hinges $(2,4)$ and fixed hinges $(1,3)$.

La géométrie de la nappe déplacée est alors calculée en utilisant P'un des deux modèles de plis décrits cidessus. Il faut noter que quand le système chevauchant est constitué par une imbrication d'écailles, la géométrie finale dépend de la séquence de fonctionnement des différents accidents (fig. 6).

Les géométries résultant de la modélisation numérique peuvent alors être comparées avec les données issues d'exemples naturels.

A

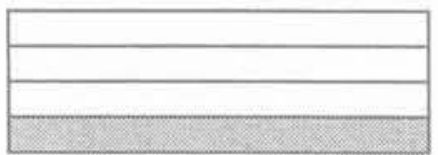

1

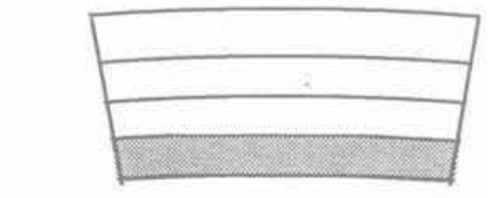

2

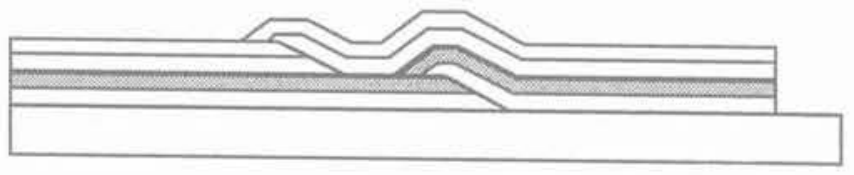

2

3

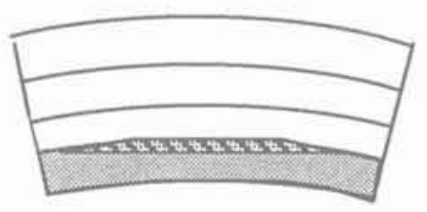

A

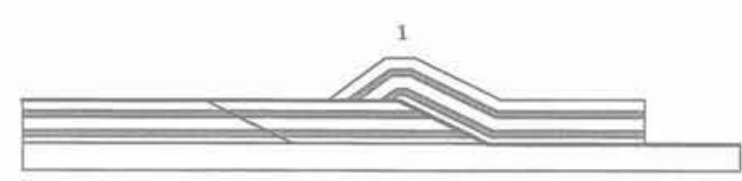

B
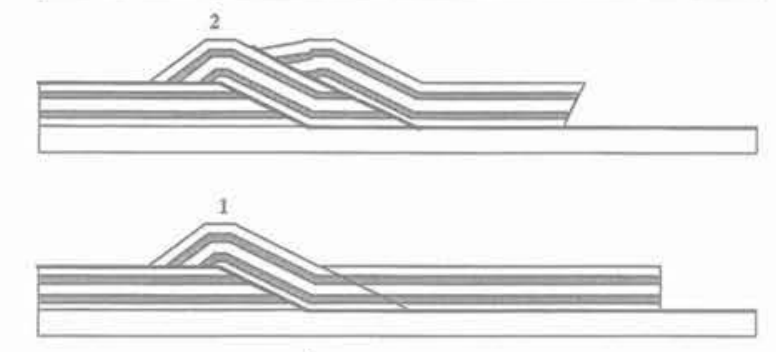

2

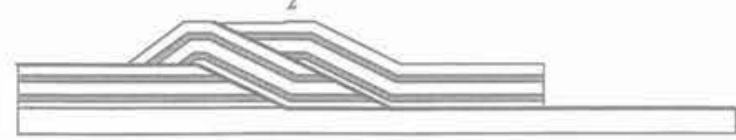

Fig. 6. - Développement d'écailles imbriquées:

A: dans une séquence prograde;

$B$ : dans une séquence rétrograde.

Fig. 6. - The development of a duplex.

$A$ : in a forward sequence, $B$ : in an hinterward sequence.

\section{APPLICATION A UN EXEMPLE GÉOLOGIQUE : LES APENNINS}

\subsection{Présentation régionale des Apennins}

La chaîne montagneuse des Apennins s'étend sur la plus grande longueur de l'Italie (fig. 7). Elle est constituée par un empilement de nappes déplacées vers l'est, en avant duquel s'est développé un bassin externe: lavant-fosse adriatique et la plaine du Pô
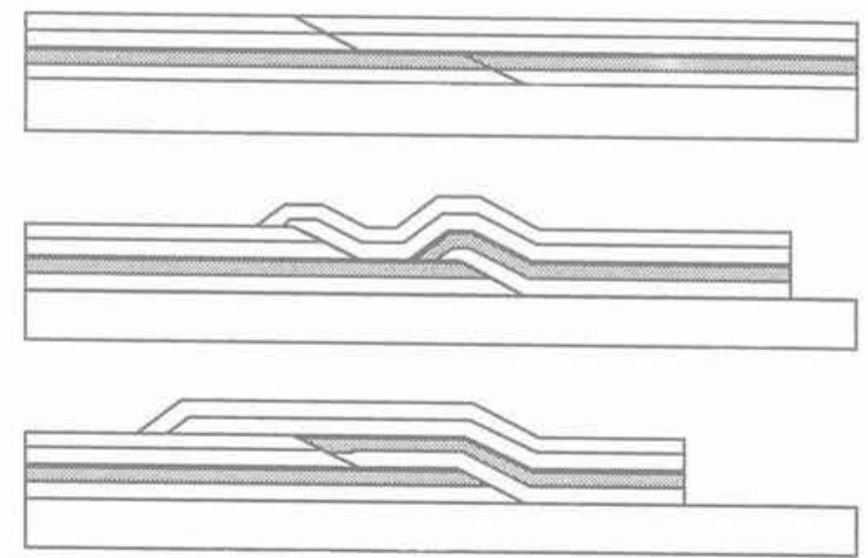

4

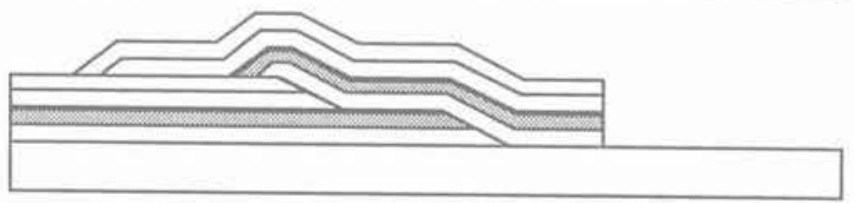

Fig. 5. - Fermeture d'un piège structural (anticlinal) pour une roche poreuse (sombre); A : plissement par flambage ; B: plissement passif au-dessus des irrégularités des surfaces de failles.

Fig. 5. - The closure of a petroleum structural trap in a porous rock (dark level); $A$ : in a buckled fold,

$B$ : in passive hanging-wall anticlines. 

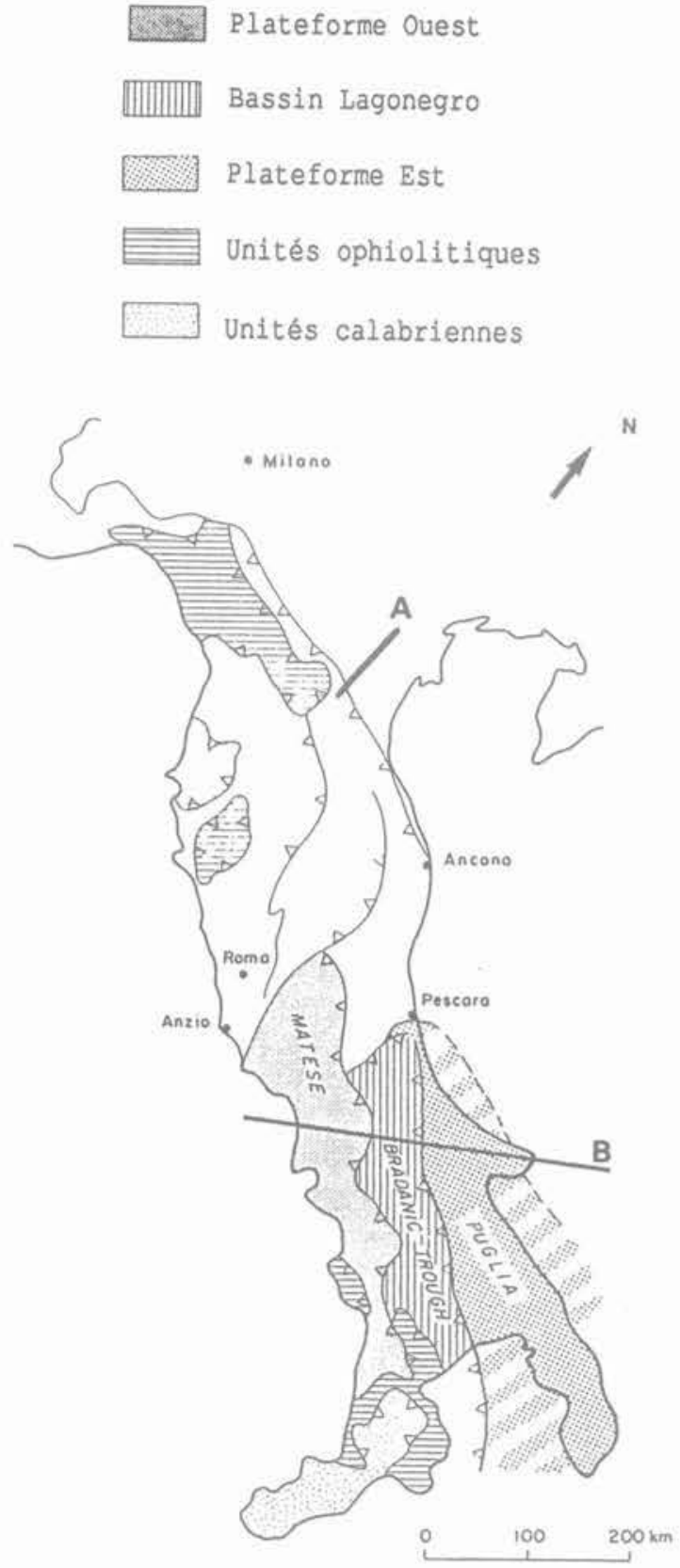

Fig. 7. - Schéma structural des Apennins et localisation des coupes des figures 8 (A) et 11 (B).

Fig. 7. - Structural map of the Apennins and cross-sections location. Figures $8(A)$ et $11(B)$.

(PAROTTO et PRATURLON, 1979). L'origine des Apennins, à l'échelle des plaques lithosphériques, est à relier à la convergence des plaques africaines et européennes (PHILIP, 1983). L'édification de la chaîne s'est réalisée de l'oligocène à la période actuelle.

\subsection{Simulation des relations entre tectonique et sédimentation}

A l'avant de la chaîne des Apennins se produisent à la fois des phénomènes tectoniques et sédimentaires. La sédimentation est commandée par la subsidence régionale de la croûte terrestre sous l'effet de la charge du relief montagneux, tandis que les chevauchements correspondent au domaine frontal de la chaîne. Ces structures sont connues grâce aux forages et aux profils de sismique réflection verticale (BALLY, 1983) (fig. 8). Elles correspondent à un empilement de multiples écailles. Le raccourcissement horizontal global absorbé dans cet empilement, et qui correspond au déplacement de l'écaille la plus interne par rapport à son autochtone, est de l'ordre de $30 \mathrm{~km}$.

Les relations géométriques entre les réflecteurs marqueurs des différentes couches sédimentaires enregistrent la compétition entre phénomènes tectoniques et phénomènes sédimentaires lors du comblement de la dépression (PION, 1987) (fig. 9). L'analyse de ces relations géométriques, et la datation des horizons stratigraphiques permet d'estimer les vitesses de déplacement sur les différentes failles. Cependant, l'interprétation des différents biseaux que l'on rencontre dans la pile sédimentaire n'est pas toujours évidente, et nous avons effectué des modélisations géométriques pour les décrypter. Sur la figure 10 on constate que les biseaux sédimentaires (appelés en anglais onlap) s'éloignent au cours du temps de la pointe de l'écaille chevauchante si la vitesse de surrection tectonique dépasse celle de la sédimentation. Les vitesses moyennes de déplacement de l'écaille la plus interne de la figure 8 ont ainsi été estimées : $0,8 \mathrm{~mm} / \mathrm{an}$ de -15 à $-5 \mathrm{MA}, 4 \mathrm{~mm} / \mathrm{an}$ de $-5 \mathrm{MA}$ à nos jours.

\subsection{Origine de l'épaississement crustal dans la zone axiale}

Dans la zone axiale de la chaîne, les données sont beaucoup moins nombreuses et de qualité moindre que dans la zone externe. Aussi plusieurs interprétations concernant les structures profondes sont envisagées. Plusieurs d'entre elles ont été testées en utilisant le programme "CICERON " (ENDIGNOUX et al., 1989 a et b). Les deux hypothèses extrêmes sont représentées sur la figure 11. Soit l'épaississement crustal n'est lié qu'à un empilement d'écailles sédimentaires, soit le coeur de l'empilement de nappes sédimentaires est constitué d'une écaille de socle. Si les deux géométries finales sont compatibles avec les données actuelles sur les structures, les vitesses de déplacement qu'elles sous-tendent peuvent servir de critère pour choisir entre les deux interprétations.

En effet, d'après les datations des sédiments syntectoniques (CASERO et al., 1989), la structuration s'est réalisée en moins de $2 \mathrm{MA}$. L'hypothèse de l'empilement de nappes sédimentaires implique donc une vitesse de déplacement à l'arrière supérieure à $30 \mathrm{~mm} / \mathrm{an}$, alors que dans l'hypothèse du socle impliqué elle est supérieure à $10 \mathrm{~mm} / \mathrm{an}$. Ces vitesses sont très rapides $\mathrm{si}$ on les compare avec les vitesses estimées pour la convergence des plaques Europe-Afrique (PLATT et al., 1989) où à celles déterminées sur la coupe de la plaine du Pô (fig. 8). Aussi l'hypothèse de l'implication du socle, qui minimise la vitesse, est préférée. 

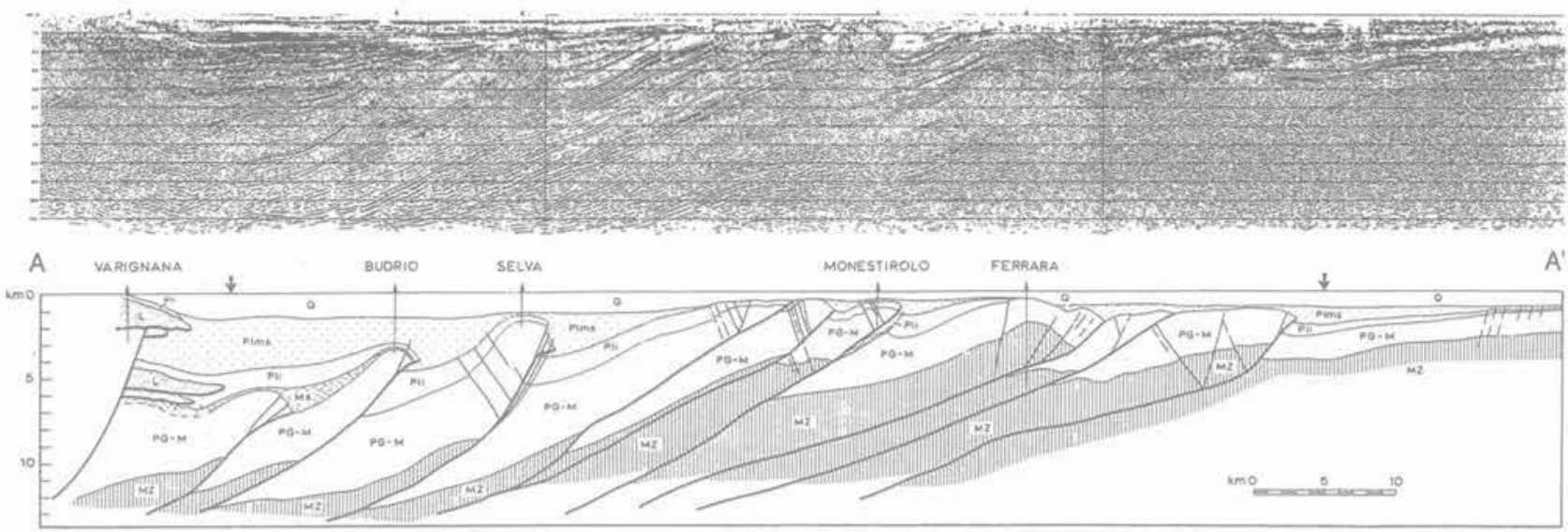

Fig. 8. - Profil sismique interprété de la zone frontale des Apennins du nord (d'après Castellarin et al., 1985 et Pion, 1987). Fig. 8. - Seismic profile of the front of the Apennins in the Pô plain.

\section{COUPLAGE \\ ENTRE MODÈLES CINÉMATIQUES ET MODÈLES PHYSIQUES}

\subsection{Flexion de la lithosphère et empilement des nappes}

Le poids des empilements de nappes affecte l'équilibre des forces quil s'exercent dans la croute terrestre.
Un modèle de flexion élastique peut, en première approximation, rendre compte de cet effet de charge (KARNER et WATTS, 1983).

Ce modèle est constitué par une plaque élastique mince représentant la lithosphère, reposant sur un milieu fluide représentant l'asthénosphère et soumise sur ses deux faces à des forces verticales liées à la gravité. La géométrie du bassin sédimentaire localisé à l'avant de l'empilement des nappes est alors fonction de la raideur de la plaque (D) et des sollicitations

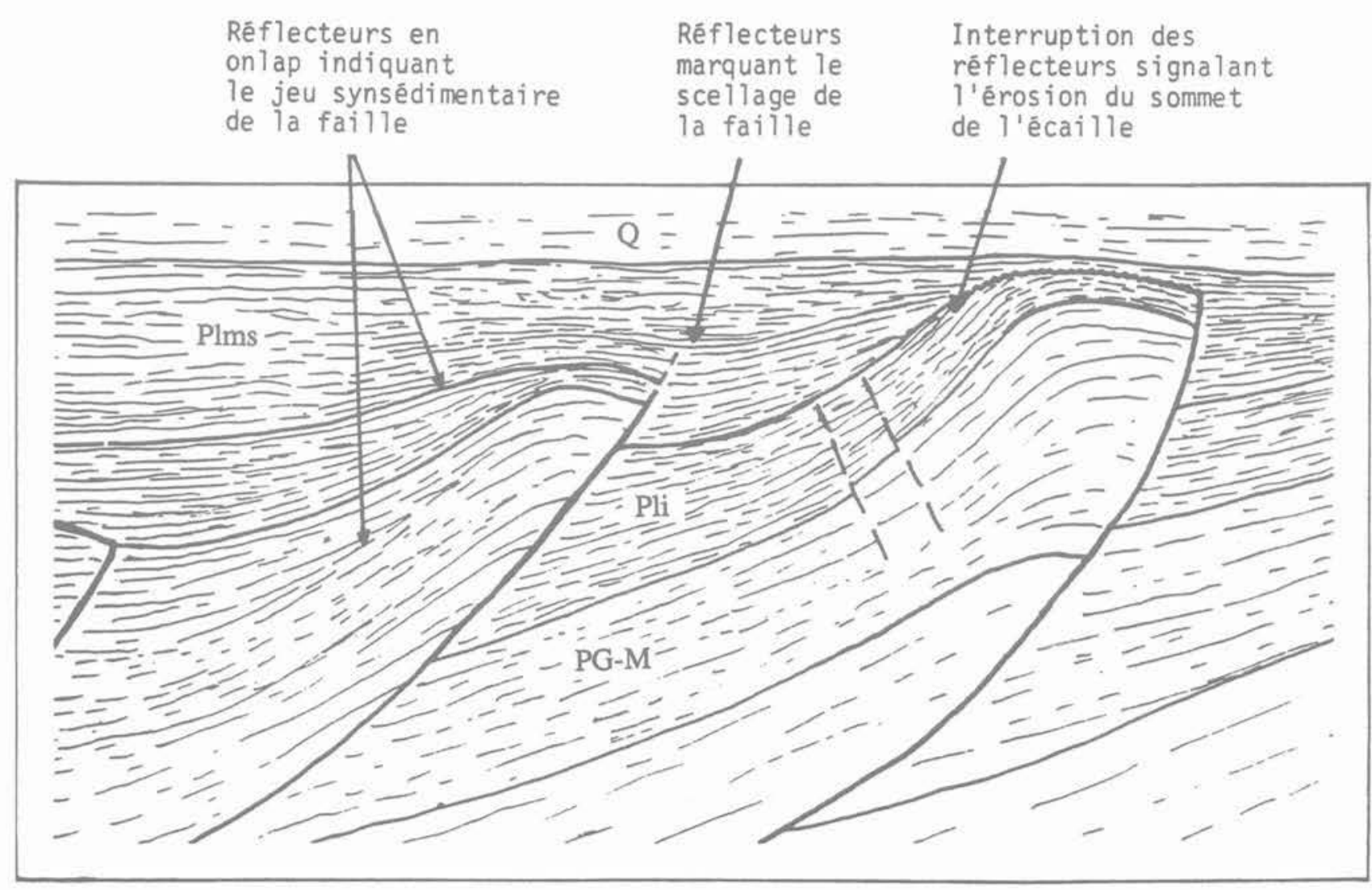

Fig. 9. - Principales organisations de réflecteurs permettant de dater le jeu d'une faille. Fig. 9. - Reflectors pattern and datation of the work of a fault. 

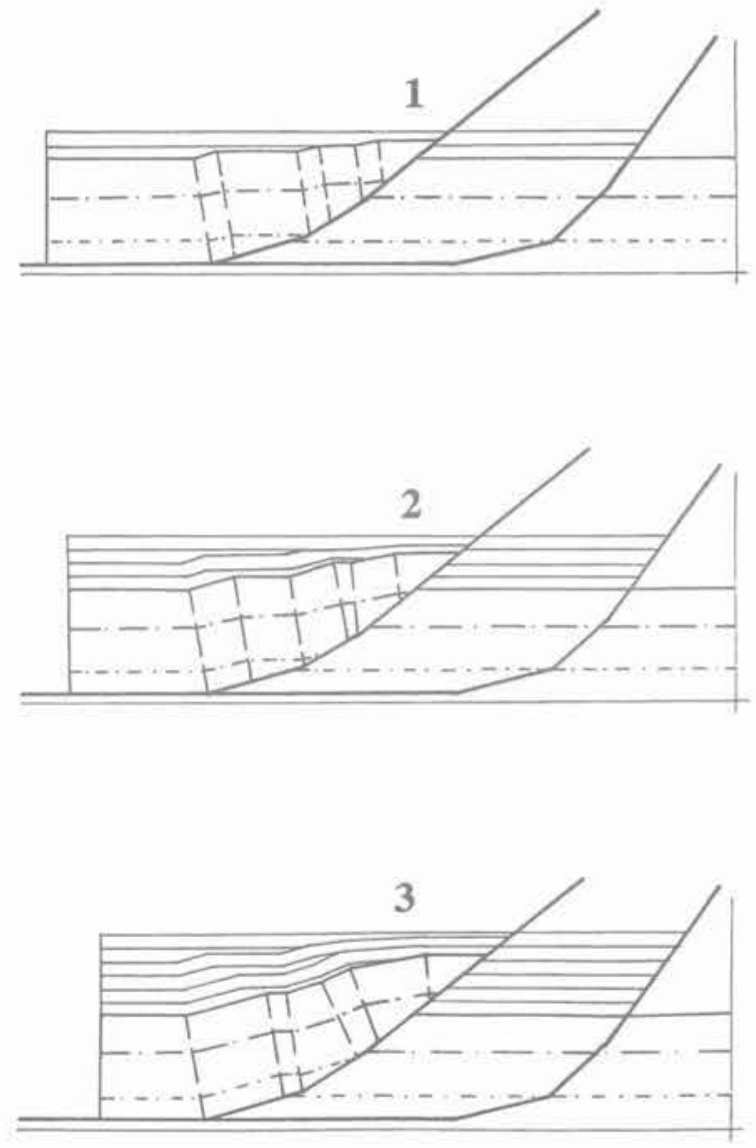

Fig. 10. - Modélisation numérique de l'évolution des bassins transportés.

Fig. 10. - Numerical modeling of «piggy-back» basins.
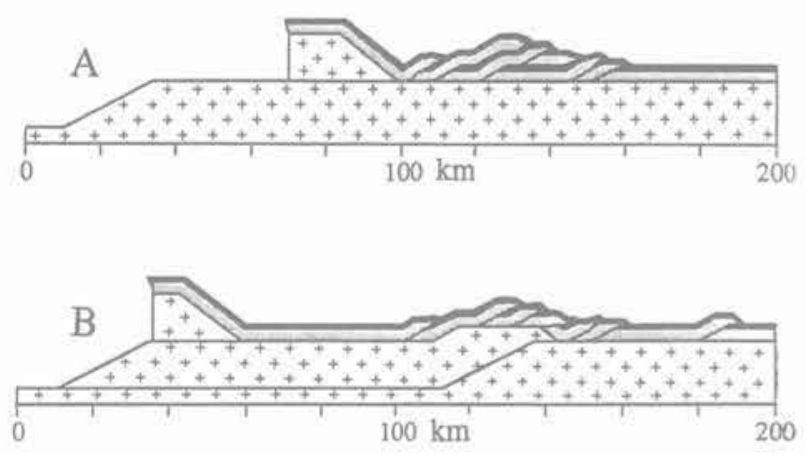

Fig. 11. - Modélisation de l'empilement des nappes le long de la coupe B. (A): empilement d'écailles sédimentaires.

(B) : écaille de socle au cceur de l'antiforme.

Fig. 11. - Numerical modeling of thrust sheets stacked along cross-section $B$.

mécaniques à son extrémité (effort tranchant Vo, Moment de flexion $\mathrm{M}$ ). Un couplage entre modèle de flexion et modèle cinématique permet de déterminer l'évolution de la forme du bassin d'avant-pays. La figure 12 illustre les résultats d'un tel couplage. Le programme de flexion utilisé est celui développé par LYON-CAEN et MOLNAR (1984). Le programme de cinématique des chevauchements est une adaptation du programme de JONES et LINSSER (1986). Ce type de couplage montre que durant le déplacement d'une écaille chevauchante crustale (ici 10 km d'épais- seur), le bassin d'avant-pays conserve un volume pratiquement constant, l'érosion qui se produit dans sa zone interne compensant la sédimentation qui se produit, elle, dans la zone plus externe. La géométrie d'ensemble du bassin varie assez peu, et elle est pour l'essentiel translatée horizontalement, en même temps que l'écaille chevauchante avance. Dès lors, si la sédimentologie montre un abrupt changement dans l'évolution du bassin, ceci implique un changement dans l'évolution de la chaîne de montagne: soit la géométrie de l'empilement de nappe évolue, soit des phénomènes tectoniques autres que la flexion se produisent, liés par exemple à des anomalies thermiques (FLEITOUT, 1990).

\subsection{Grands déplacements}

dans un modèle thermique en éléments finis Les modèles cinématiques permettent de simuler de grands déplacements et ainsi de transporter des maillages sur lesquels sont réalisés des calculs portant sur des grandeurs physiques (SHI et WANG, 1987 ; ENDIGNOUX et WOLF, 1990).

Sur la figure 13, P'évolution thermique d'une structure anticlinale a été modélisée en éléments finis,
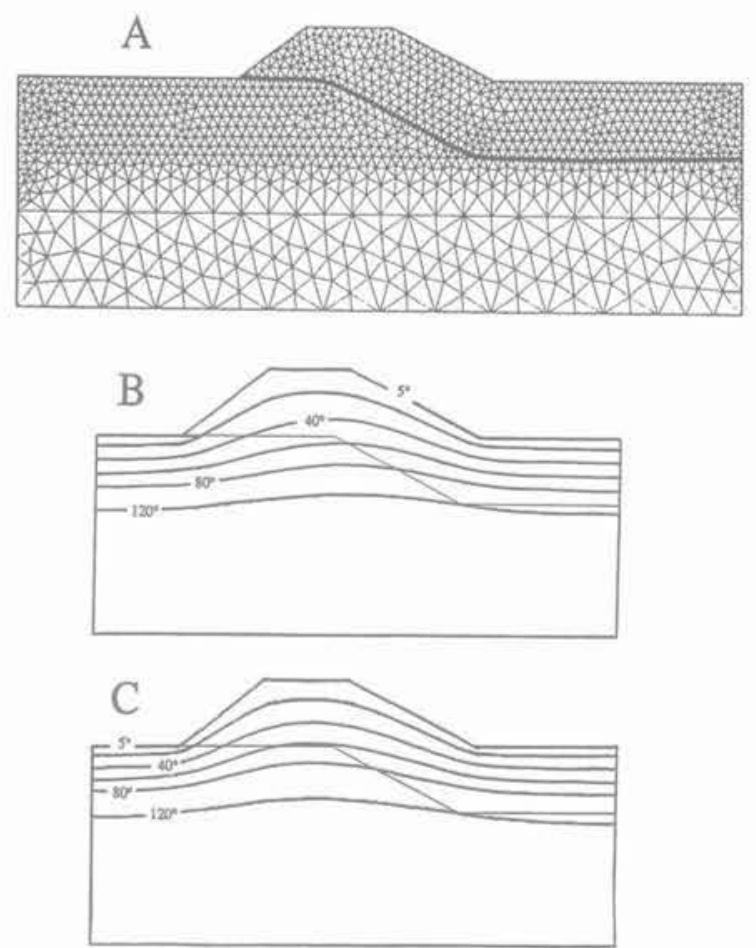

Fig. 13. - Modélisation de l'évolution thermique d'une structure chevauchante.

A : maillage en eléments finis utilisé. B : situation thermique de la structure juste à la fin du chevauchement. Les isothermes sont représentés en trait gras (masse volumique : 2100 $\mathrm{kg} / \mathrm{m}^{3}$; chaleur massique : $1000 \mathrm{~J} / \mathrm{kg}^{\circ} \mathrm{K}$; conductivité thermique : $2,1 \mathrm{~W} / \mathrm{m}^{\circ} \mathrm{K}$; épaisseur du chevauchement : $4 \mathrm{~km}$ : vitesse de chevauchement : $1 \mathrm{~cm} / \mathrm{an}$; déplacement total le long du chevauchement: $12 \mathrm{~km}$. C : régime thermique permanent de la structure.

Fig. 13. - numerical modelling of the thermal evolution of a thrust sheet. A : finite element mesh. B: thermal evolution of a thrust sheet after $0.6 \mathrm{Mr}$. (density: $2100 \mathrm{~kg} / \mathrm{m}^{3}$; heat capacity : $1000 \mathrm{~J} / \mathrm{kg}^{\circ} \mathrm{K}$; conductivity; $2.1 \mathrm{~W} / \mathrm{m}^{\circ} \mathrm{K}$; sheet thickness: $4 \mathrm{~km}$; thrust rate: $1 \mathrm{~cm} / \mathrm{an}) . \mathrm{C}$ : steady state. 

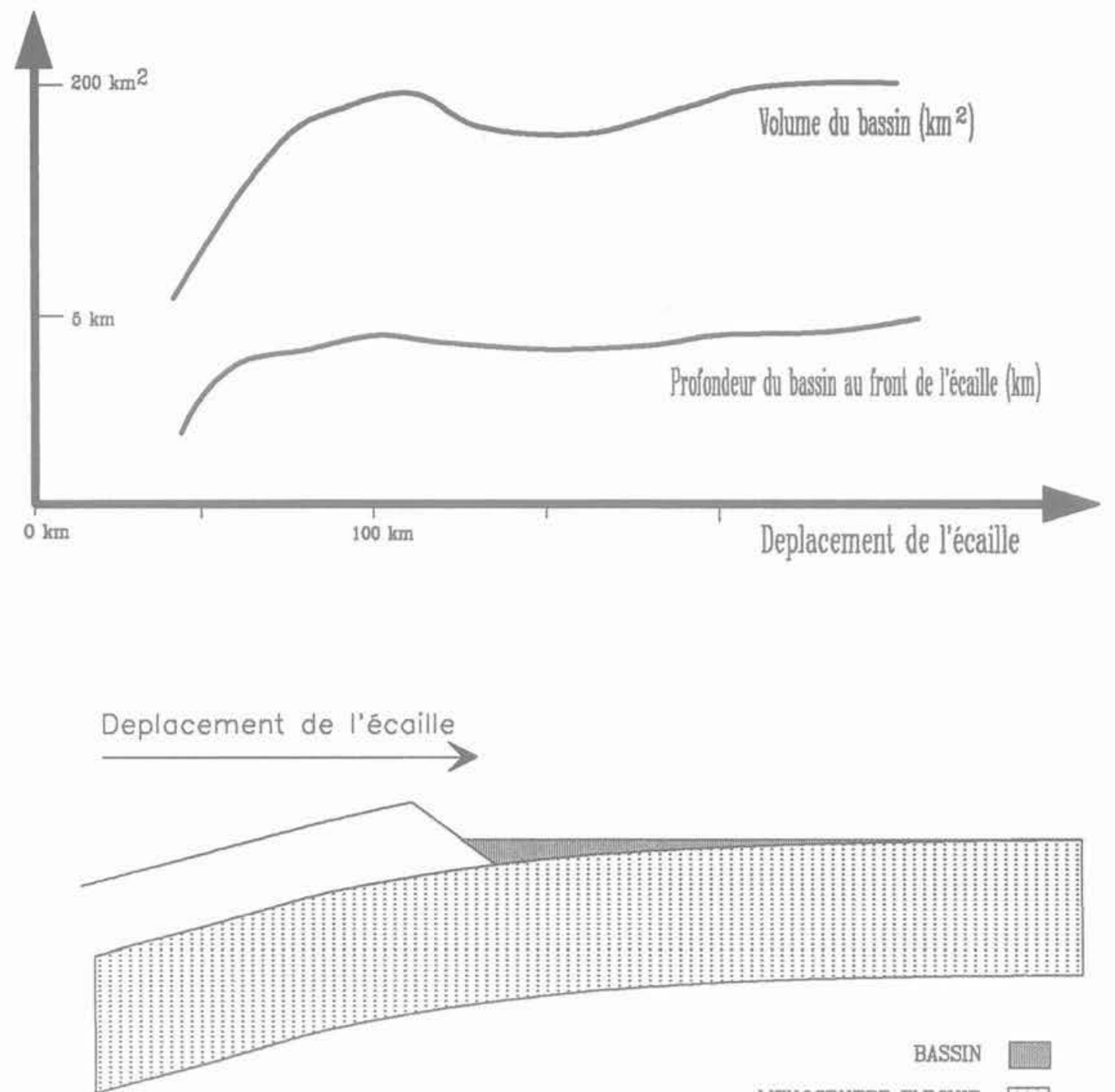

\section{LITHOSPHERE FLECHIE}

ECALLES CHEVAUCHANTES

Fig. 12. - Modélisation de l'évolution de la forme d'un bassin d'avant-pays A : modèle de flexion

favec raideur de la plaque $D=8.10^{22} \mathrm{~N} . \mathrm{m}$; densité du bassin $: 2,4$; densité de l'écaille chevauchante 2,8 ;

densité du manteau fluide sous-jacent à la lithosphère fléchie: 3,$3 ; B$ : évolution du volume et de la profondeur du bassin en fonction du déplacement de l'écaille.

Fig. 12. - Numerical modeling of a foreland basin. A : flexural model (stiffness $D=810^{22} \mathrm{~N} \mathrm{~m}$; density of the basin : 2.4 ; density of the thrust sheet: 2.8 ; density of the mantle: 3.3 )

$B:$ the volume and the depth of the basin versus the displacement of the load.

grâce à un couplage entre le mailleur MODULEF et le programme CICERON. Ce type de modélisation montre que pour des écailles chevauchantes d'épaisseur inférieure à $10 \mathrm{~km}$, les vitesses de chevauchement sont suffisamment lentes $(<1 \mathrm{~cm} / \mathrm{an})$ pour que le régime thermique transitoire durant la déformation soit peu éloigné, quoique un peu plus froid, du régime thermique permanent qui suit la fin des déplacements.

\subsection{Signification physique d'une discontinuité cinématique}

Ce type de couplage entre modèle cinématique et modèle thermique conduit à s'interroger sur la signi- fication physique d'une discontinuité cinématique à la base d'une nappe. En effet, du point de vue numérique, les vitesses et les températures sont interpolées au travers de la faille de manière à assurer la continuité de la température dans un milieu solide conducteur de la chaleur (fig. 14).

La faille, en tant qu'objet géologique (fig. 15) n'est pas simplement l'expression du déplacement de deux blocs rigides. Les observations géologiques montrent que dans la croûte inférieure, à plus de $10 \mathrm{~km}$ de profondeur, une faille est en fait une zone de cisaillement très intense, où la déformation est continue. Par contre, dans la croûte supérieure, la faille et son voisinage est en fait un système thermodynamique composé de fluides et de solides, avec des change- 


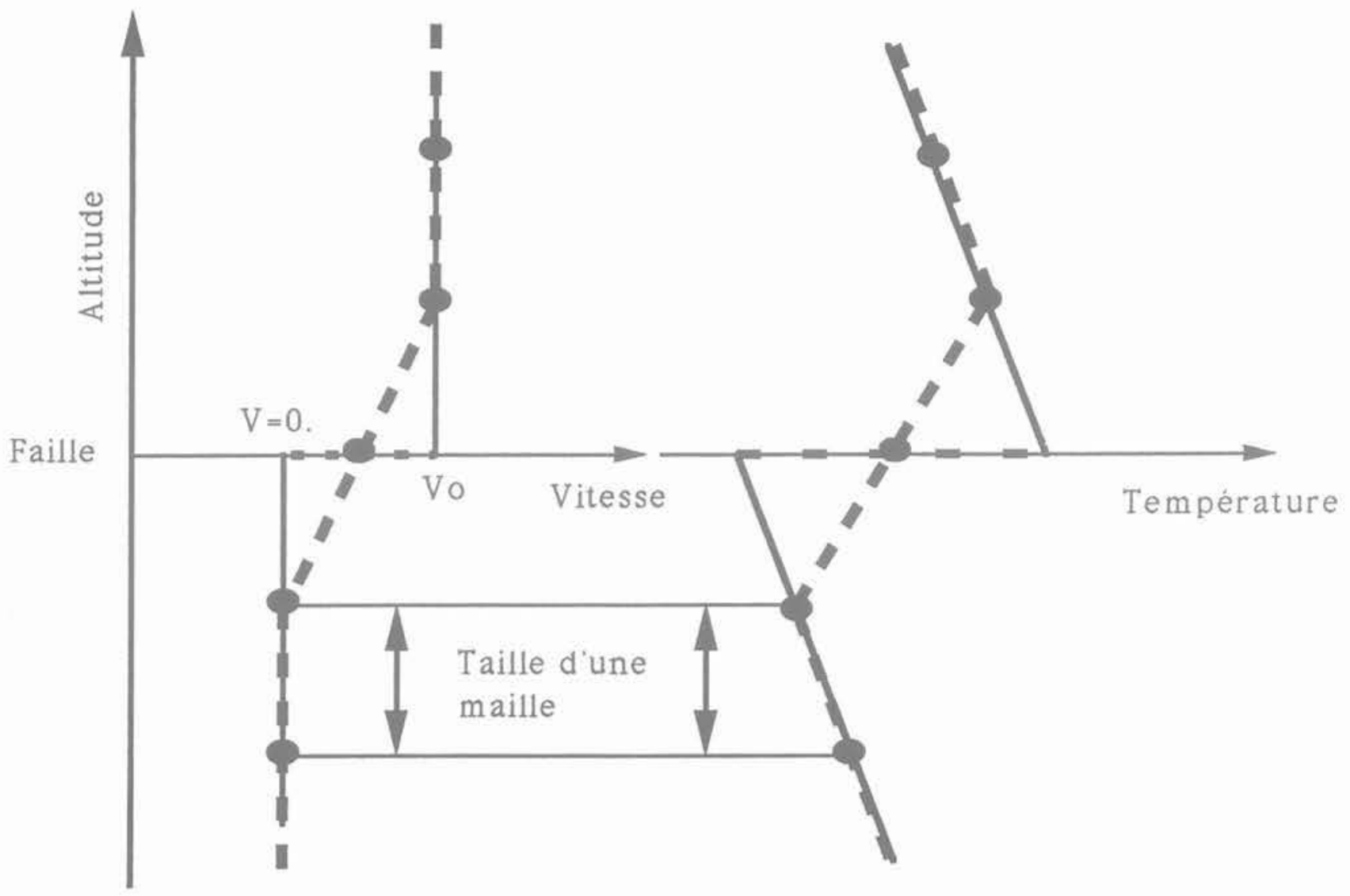

Champ de vitesse et de température après déplacement

$-2=$ Champ de vitesse et de température après interpolation et position du noeuds du maillage

Fig. 14. - Modèle numérique de discontinuité cinématique. Fig. 14. - The numerical modeling of a kinematical discontinuity.

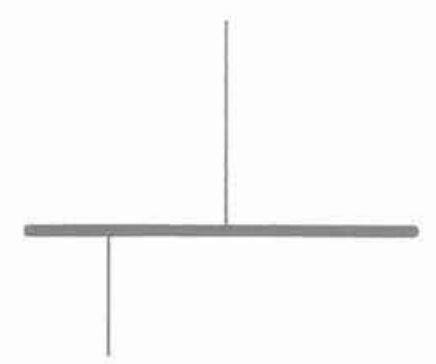

DEPLACEMENT DE BLOCS RIGIDES

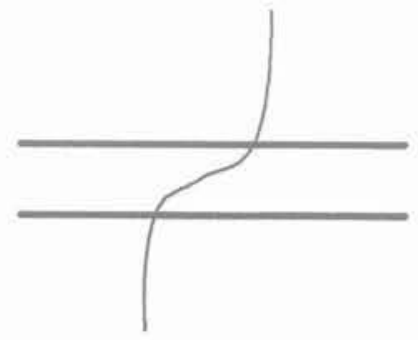

ZONE DE CISAILLEMENT

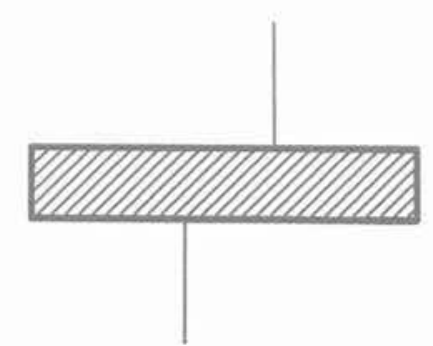

SYSTEME THERMODYNAMIQUE FLUIDE+SOLIDE AVEC CHANGEMENT DE PHASES

Fig. 15. - Interprétation d'une faille géologique.

Fig. 15. - The interprertation of a geological fault. 
ments de phase (VIALON, 1990). Dès lors, la cinématique des milieux solides ne permet pas une description des phénomènes physiques présents au voisinage d'une faille, et la prise en compte des fluides doit être envisagée aussi bien du point de vue transfert thermique (chute ou augmentation de la conductivité ?) que du point de vue mécanisme de la déformation.

\section{CONCLUSION}

Les procédures de modélisation basées sur des algorithmes cinématiques directs permettent de simuler les très grands déplacements observés par les géologues, même s'ils ne rendent pas compte dans le détail de la complexité des structures géologiques. Ils fournissent donc un outil permettant de tenir compte des grands déplacements dans l'étude physique des mécanismes tectoniques.

Les vitesses de déplacement qui ont pu être déterminées pour les nappes sont faibles, de l'ordre de 1 à $10 \mathrm{~mm} / a n$. Aussi les structures en cours de déplacement ont un régime thermique assez proche du régime permanent et les structures tectoniques sont à tout instant en équilibre isostatique. Les phénomènes d'érosion et de sédimentation se produisent durant le déplacement de la nappe avec des vitesses du même ordre de grandeur et influencent considérablement l'évolution tectonique des nappes.

\section{REMERCIEMENTS}

Nous remercions l'Institut Français du Pétrole pour le support financier apporté à ce travail dans le cadre d'une convention de recherche avec l'Université Joseph-Fourier de Grenoble (convention $n^{\circ} 11632$ ).

\section{BIBLIOGRAPHIE}

BOYER S.E., ELLIOTT D. (1982), The geometry of thrust systems. Bull. Am. Ass. Pet. Geol., 66, $1196-1230$

CASERO P., ROURE F., ENDIGNOUX L., MORETTI I., MULLER C., SAGE L., VIALLY R. (1989), Neogene geodynamic evolution of the Southern Apennines. Mem. Soc, Géol. It.

CASTELLARIN A., EVA C., GIGLIA G., BATTISTA VAI G. (1985),, Analisi strutturale del fronte Appenninico Padano. Giornale di Geologia, 47. p. $47-75$

CRANE R.C. (1987), Use of fault cut-offs and bed travel distance in balanced cross-sections. J. Struct. Geol,, 9, p. 243-247.

ENDIGNOUX L., MORETTI 1., ROURE F. (1989 a), Forward modeling of Southern Apennines. Tectonics, p. 1095-1104.
ENDIGNOUX L., MUGNIER J.L., (1990 b), The use of a forward kinematic model in the construction of a balanced cross-section. Tectonics, p. 1249-1262.

ENDIGNOUX L., WOLF S. (1989), Thermal and kinematic evolution of thrust basins : $2 D$ numerical model. in press. In : Petroleum tectonics in mobile belts, J. Letouzey ed., Technip Editions.

FLEITOUT L. (1990), Origine des contraintes tectoniques dans la lithosphère terrestre. Revue Française de Géotechnique (dans le présent numéro).

JONES P.B., LINSSER H. (1986), Computer synthesis of balanced structural cross-sectrion by forward modelling. Am. Assoc. Pet. Geol. Bull., 70 (5), (Abstract), p. 605.

KARNER G.D., WATTS A.B. (1983), Gravity anomalies and flexure of the lithosphere. Journal of Geophysical Research, Vol. 88, No B 12, p. 445-477.

LYON-CAEN H., MOLNAR P. (1983), Constraints on the structure of the Himalaya from an analysis of gravity anomalies and a flexural model of the lithosphere. J. Geophys. Res., 88, p. 8171-8191.

MORETTI I., TRIBOULET S. (1990), Some remarks on the geometrical modeling of geological deformations. In : Petroleum Tectonics in mobile belts, edited by J. Letouzey, Technip Editions.

MUGNIER J.L. (1984), Déplacement et déformation dans l'avant-pays d'une chaîne de collision. Méthode d'étude et modélisation, exemple du Jura. Thèse USMG Grenoble, IRIGM, 163 p.

PION E. (1987), Evolution cinématique d'un bassin d'avant-pays. Exemple du Bassin Padan. Rapport de DEA de Mécanique des milieux géophysiques et environnement. USTM Grenoble, 125 p.

PHILIP H. (1983), La tectonique actuelle et récente dans le domaine méditerranéen et ses bordures, ses relations avec la sismicité. Thèse Montpellier.

PLATT J.P., BEHRMANN J.H., CUNNINGHAM P.C. DERVEY J.F HELMAN M., PARISH M. SHEPLEY M.G., WALLIS S., WESTON P.J. (1989), Kinematics of the Alpine arc and the motion history of Adria, Nature, 337, p. 158-161.

SHI Y., WANG C.Y. (1987), Two dimensional modeling of the PTt paths of regional metamorphism in simple overthrust terrains. Geology, 15 , p. $1048-1051$.

SUPPE J. (1983), Geometry and kinematics of faultben folding. Am. Jour, of Science, 283, p. 684-721.

SUPPE J. (1984), Principles of structural geology. New-Jersey, Prentice Hall Inc., 300 p.

VIALON P. (1990), Objet et démarche de la tectonique. Revue Française de Géotechnique (dans le présent numéro). 